\title{
A Spectroscopic Study of the Solar Corona from Norikura and SOHO data
}

\author{
K. P. Raju \\ Indian Institute of Astrophysics, Koramangala, Bangalore-560 034, \\ India \\ T. Sakurai and K. Ichimoto \\ National Astronomical Observatory of Japan, 2-21-1, Ohsawa, Mitaka, \\ Tokyo-181, Japan
}

\begin{abstract}
We report the results from a spectroscopic study of the solar corona using both ground-based and space-based data. Some of the current topics in coronal physics, such as the plume-interplume differences in coronal holes and wave propagation in the corona have been examined. The distribution of emission line intensities, Doppler velocities and line widths were obtained from the spectroscopic observations from Norikura Solar Observatory of National Astronomical Observatory of Japan. The coronal images in Fe IX,X $171 \AA$ and Fe XII $195 \AA$ from SOHO EIT were used to get the temperature map of the corona. Combining both results, we have obtained the nonthermal velocities in the coronal hole region, without the usual assumption of a uniform ion temperature. It has been found that the histogram distributions of Doppler velocities and nonthermal velocities for coronal hole and quiet regions are markedly different. The nonthermal velocities have been found to be larger by about $27 \%$ at the interplume regions as compared to plumes which supports the view that the interplume regions are the source regions of the fast solar wind. The analysis of the time sequence spectral data shows signatures of coronal oscillations at localized regions. Periods of the order of a few minutes have been found mainly in Doppler velocities.
\end{abstract}

\section{Introduction}

The space missions in recent years have given unprecedented insights to coronal physics. The high resolution data from SOHO, YOHKOH and TRACE have been used to study the fundamental problems such as coronal heating and the acceleration of the fast solar wind (Domingo, Fleck, \& Poland 1995). The present study uses both space-based and ground-based data to examine some of the specific aspects related to the above problems such as the physical characteristics of coronal holes, differences between plumes and interplumes, and coronal oscillations.

It is well-known that the high speed solar wind originates from coronal holes (Krieger, Timothy, \& Roelof 1973). However, there has been a debate on the exact source regions of the fast solar wind in coronal holes, i.e., whether it 
emanates from plumes or interplume regions (Hassler et al. 1997). This has come up from a recent finding that the emission line widths in plumes are less than that in the nearby interplume regions (Wilhelm et al. 1998). We have examined the differences in physical quantities in coronal holes and quiet regions on one hand and plumes and interplumes on the other hand. A specific advantage with our method is that the usual assumption of a uniform ion temperature is not involved.

There have been some reports of coronal oscillations with periods of the order of several minutes. Tsubaki (1987) lists various studies of coronal oscillations based on eclipse or coronagraphic observations. The oscillations are seen in the intensity, Doppler velocity and/or in the width of coronal emission lines. There have also been some recent reports of coronal/transition region oscillations from SOHO (Ofman, Nakariakov and DeForest 1999, Fludra 1999). Brynildsen et al. (1999) analysed SOHO SUMER data and have found $3 \mathrm{~min}$ oscillations in intensity, velocity and width of transition region lines. By using a coronagraph, we have obtainted time sequences of coronal spectra for the duration of a few hours with cadence varying from 12 to $48 \mathrm{sec}$. The power spectral analysis of the data show evidence of coronal oscillations with periods of several minutes.

\section{Results}

The details of the instrument, observation and analysis are described in Ichimoto et al. (1995), Singh et al. (1999) and Raju et al. (2000). The histogram distribution of Doppler velocities and nonthermal velocities in coronal hole and quiet regions obtained from the coronal red line observations are given in Figure 1. It can be seen that the magnitudes of Doppler velocities are higher in the coronal holes which is due to the enhanced outflows expected in the region. The typical nonthermal velocity in coronal holes is $24 \mathrm{~km} \mathrm{~s}^{-1}$ while that in quiet region is $15 \mathrm{~km} \mathrm{~s}^{-1}$. It has been found that the Doppler velocity shows a dip at the plume-center. The typical nonthermal velocity in plume is $19 \mathrm{~km} \mathrm{~s}^{-1}$ while that in interplume is $26 \mathrm{~km} \mathrm{~s}^{-1}$ (Raju et al. 2000). The enhanced Doppler velocities and nonthermal velocities in the interplume regions suggest that they are the source regions of the fast solar wind.

Time sequences of intensity, Doppler velocity and line width from a point in the western limb obtained from coronal green line observations are shown in Figure 2. The corresponding power spectra are shown in the right panel. It may be seen that there are some significant periodicities around 4-6 minutes which is especially seen in Doppler velocity. The result generally agrees with the findings of Tsubaki (1987), and the nature and causes of these oscillations are under investigation.

\section{References}

Brynildsen, N., Kjeldseth-Moe, K., Maltby, P., \& Wilhelm, K. 1999, ApJ, 517, L159

Domingo, V., Fleck, B. \& Poland, A.I., 1995, Sol. Phys.,162, 1.

Fludra, A., 1999, A\&A, 344, L75 

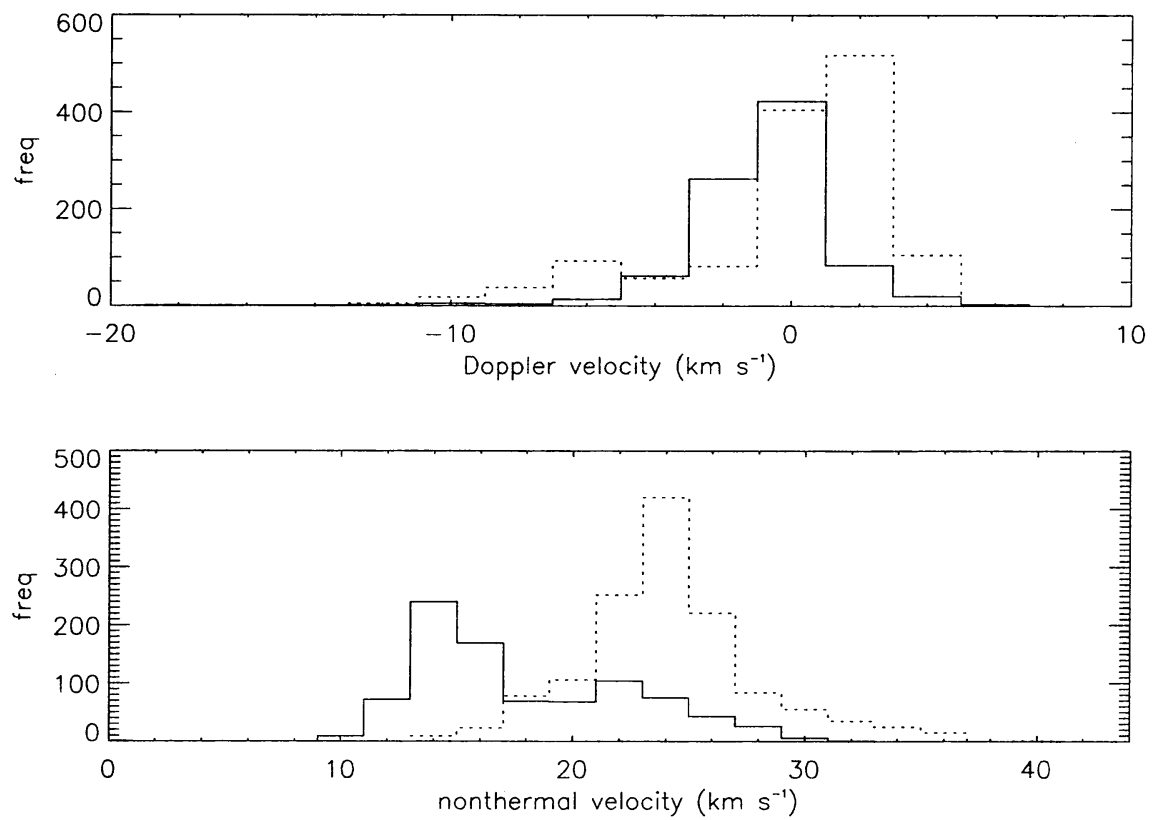

Figure 1. Histograms of Doppler velocity and nonthermal velocity from the coronal red line observations. The dotted line represents the coronal hole, and the solid line represents the quiet region outside. The Doppler velocities are with respect to the coronal rest frame.

Hassler, D.M., Wilhelm, K., Lemaire, P., Schuhle, U. 1997, Sol. Phys., 175, 375 Ichimoto, K., Hara, H., Takeda, A., Kumagai, K., Sakurai, T., Shimizu, T., \& Hudson, H.S. 1995, ApJ, 445, 978

Krieger, A.S., Timothy, A.F., \& Roelof, E.C. 1973, Sol. Phys., 49, 43 Ofman, L., Nakariakov, V.M., \& DeForest, C.E., 1999, ApJ, 514, 441 Raju, K.P., Sakurai, T., Ichimoto, K., \& Singh, J. 2000, ApJ, in press Singh, J., Ichimoto, K., Imai, H., Sakurai, T., \& Takeda, A. 1999, PASJ, 51,269 Tsubaki, T., 1987, Proceedings of 9th Sac Peak Workshop, Altrock (ed), p.140 Wilhelm, K., Marsch, E., Dwivedi, B.N., Hassler, D.M., Lemaire, P., Gabriel A.H., \& Huber, C.E.M., 1998, ApJ, 500, 1023 

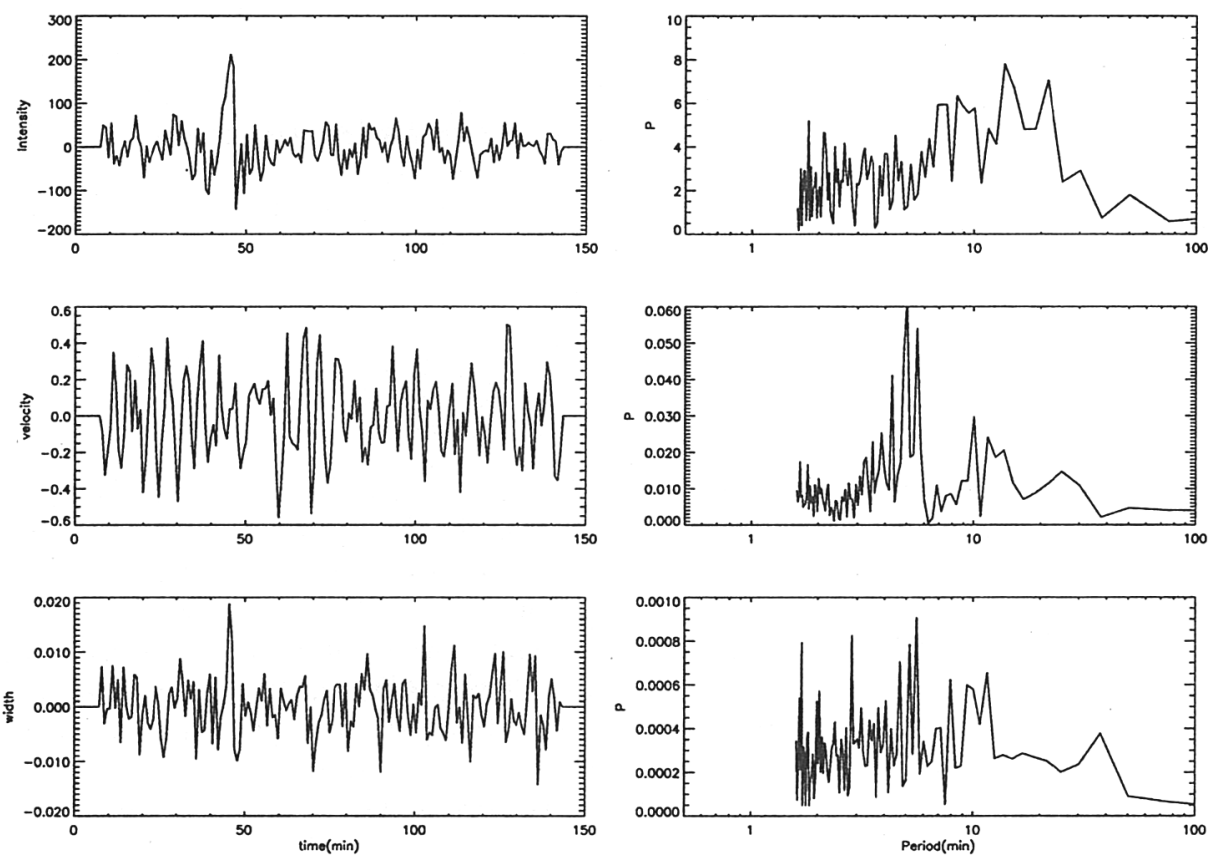

Figure 2. Time sequences of the quantities from the coronal green line spectra after subtracting out the long-term trend are given in the left panel and the corresponding power spectra are given in the right panel. 